\title{
SEARCH FOR GAMMA-RAY EMISSION FROM DES DWARF SPHEROIDAL GALAXY CANDIDATES WITH FERMI-LAT DATA
}

A. Drlica-Wagner ${ }^{1}$, A. Albert ${ }^{2}$, K. Bechtol ${ }^{3}$, M. Wood ${ }^{2}$, L. Strigari ${ }^{4}$, M. SÁnchez-Conde ${ }^{5,6}$, L. Baldini ${ }^{7}$, R. Essig ${ }^{8}$, J. Cohen-Tanugi ${ }^{9}$, B. Anderson ${ }^{66}$, R. Bellazzini ${ }^{10}$, E. D. Bloom ${ }^{2}$, R. Caputo ${ }^{11}$, C. Cecchi ${ }^{12,13}$, E. Charles ${ }^{2}$, J. Chiang ${ }^{2}$, A. De Angelis ${ }^{14}$, S. FunK ${ }^{2}$, P. Fusco ${ }^{15,16}$, F. Gargano ${ }^{16}$, N. Giglietto ${ }^{15,16}$, F. Giordano ${ }^{15,16}$, S. Guiriec ${ }^{17,67}$,

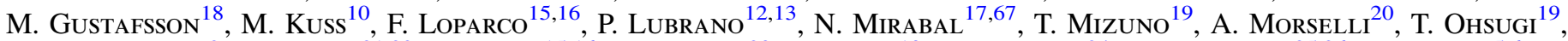
E. Orlando ${ }^{2}$, M. Persic ${ }^{21,22}$, S. Rainò ${ }^{15,16}$, N. SehGal ${ }^{23}$, F. Spada ${ }^{10}$, D. J. Suson ${ }^{24}$, G. Zaharijas ${ }^{25,26}$, S. Zimmer ${ }^{5,6}$

(The Fermi-LAT Collaboration),

T. Abbott $^{27}$, S. Allam ${ }^{1,28}$, E. Balbinot ${ }^{29,30}$, A. H. Bauer ${ }^{31}$, A. Benoit-Lévy ${ }^{32}$, R. A. Bernstein ${ }^{33}$, G. M. Bernstein ${ }^{34}$, E. Bertin ${ }^{35}$, D. Brooks ${ }^{32}$, E. Buckley-Geer ${ }^{1}$, D. L. Burke ${ }^{36}$, A. Carnero Rosell ${ }^{30,37}$, F. J. Castander ${ }^{31}$, R. Covarrubias ${ }^{38}$, C. B. D'Andrea ${ }^{39}$, L. N. DA Costa ${ }^{30,37}$, D. L. DePor ${ }^{40}$, S. Desai ${ }^{41,42}$, H. T. Diehl ${ }^{1}$, C. E. Cunha ${ }^{43}$, T. F. Eifler ${ }^{34,44}$, J. Estrada ${ }^{1}$, A. E. Evrard ${ }^{45}$, A. Fausti Neto ${ }^{30}$, E. Fernandez ${ }^{46,47}$, D. A. Finley ${ }^{1}$, B. Flaugher ${ }^{1}$, J. Frieman ${ }^{1,3}$, E. Gaztanaga ${ }^{31}$, D. Gerdes ${ }^{45}$, D. Gruen ${ }^{48,49}$, R. A. Gruendi ${ }^{38,50}$, G. Gutierrez ${ }^{1}$, K. Honscheid ${ }^{51,52}$, B. Jain ${ }^{34}$, D. James ${ }^{27}$, T. Jeltema ${ }^{53}$, S. Kent ${ }^{1}$, R. Kron ${ }^{3}$, K. KuehN ${ }^{54,55}$, N. Kuropatkin ${ }^{1}$, O. Lahav ${ }^{32}$, T. S. Li ${ }^{40}$, E. Luque ${ }^{56}$, M. A. G. Maia ${ }^{30,37}$, M. MaKler ${ }^{57}$, M. March ${ }^{34}$, J. Marshall ${ }^{40}$, P. Martini ${ }^{51,58}$, K. W. Merritt ${ }^{1}$, C. Miller ${ }^{45,59}$, R. Miquel ${ }^{46,47}$, J. Mohr ${ }^{41,42}$, E. Neilsen ${ }^{1}$, B. Nord ${ }^{1}$, R. Ogando ${ }^{30,37}$, J. Peoples ${ }^{1}$, D. Petravick ${ }^{38}$, A. Pieres ${ }^{56}$, A. A. Plazas ${ }^{44,60}$, A. Queiroz ${ }^{56}$,

A. K. Romer ${ }^{61}$, A. Roodman ${ }^{36,43}$, E. S. RykofF ${ }^{36}$, M. SaKo ${ }^{34}$, E. SAnChez ${ }^{62}$, B. Santiago ${ }^{56}$, V. Scarpine ${ }^{1}$, M. Schubnell ${ }^{45}$, I. Sevilla ${ }^{50,62}$, R. C. Smith ${ }^{27}$, M. Soares-Santos ${ }^{1}$, F. Sobreira ${ }^{1,30}$, E. Suchyta ${ }^{51,52}$, M. E. C. Swanson ${ }^{38}$, G. Tarle ${ }^{45}$,

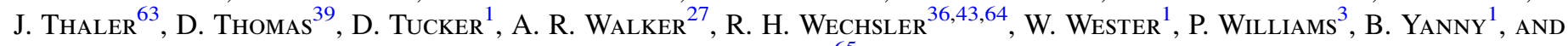
J. ZunTZ ${ }^{65}$

\section{(The DES Collaboration)}

${ }^{1}$ Center for Particle Astrophysics, Fermi National Accelerator Laboratory, Batavia, IL 60510, USA; kadrlica@ fnal.gov

${ }^{2}$ W. W. Hansen Experimental Physics Laboratory, Kavli Institute for Particle Astrophysics and Cosmology, Department of Physics and SLAC National Accelerator Laboratory, Stanford University, Stanford, CA 94305, USA; aalbert@slac.stanford.edu, mdwood@slac.stanford.edu

${ }^{3}$ Kavli Institute for Cosmological Physics, University of Chicago, Chicago, IL 60637, USA; bechtol@kicp.uchicago.edu

${ }^{4}$ Department of Physics and Astronomy, Texas A\&M University, College Station, TX 77843-4242, USA; strigari@physics.tamu.edu

5 The Oskar Klein Centre for Cosmoparticle Physics, AlbaNova, SE-106 91 Stockholm, Sweden

${ }^{6}$ Department of Physics, Stockholm University, AlbaNova, SE-106 91 Stockholm, Sweden

${ }^{7}$ Università di Pisa and Istituto Nazionale di Fisica Nucleare, Sezione di Pisa, I-56127 Pisa, Italy

${ }^{8}$ C. N. Yang Institute for Theoretical Physics, State University of New York, Stony Brook, NY 11794-3840, USA

${ }^{9}$ Laboratoire Univers et Particules de Montpellier, Université Montpellier 2, CNRS/IN2P3, Montpellier, France ${ }^{10}$ Istituto Nazionale di Fisica Nucleare, Sezione di Pisa, I-56127 Pisa, Italy

${ }^{11}$ Santa Cruz Institute for Particle Physics, Department of Physics and Department of Astronomy and Astrophysics, University of California at Santa Cruz, Santa Cruz, CA 95064, USA

12 Istituto Nazionale di Fisica Nucleare, Sezione di Perugia, I-06123 Perugia, Italy

${ }^{13}$ Dipartimento di Fisica, Università degli Studi di Perugia, I-06123 Perugia, Italy

${ }^{14}$ Dipartimento di Fisica, Università di Udine and Istituto Nazionale di Fisica Nucleare, Sezione di Trieste, Gruppo Collegato di Udine, I-33100 Udine, Italy

${ }^{15}$ Dipartimento di Fisica "M. Merlin" dell’Università e del Politecnico di Bari, I-70126 Bari, Italy

${ }^{16}$ Istituto Nazionale di Fisica Nucleare, Sezione di Bari, I-70126 Bari, Italy

${ }^{17}$ NASA Goddard Space Flight Center, Greenbelt, MD 20771, USA

${ }^{18}$ Georg-August University Göttingen, Institute for theoretical Physics-Faculty of Physics, Friedrich-Hund-Platz 1, D-37077 Göttingen, Germany

${ }^{19}$ Hiroshima Astrophysical Science Center, Hiroshima University, Higashi-Hiroshima, Hiroshima 739-8526, Japan

${ }^{20}$ Istituto Nazionale di Fisica Nucleare, Sezione di Roma "Tor Vergata", I-00133 Roma, Italy

${ }^{21}$ Istituto Nazionale di Fisica Nucleare, Sezione di Trieste, I-34127 Trieste, Italy

22 Osservatorio Astronomico di Trieste, Istituto Nazionale di Astrofisica, I-34143 Trieste, Italy

${ }^{23}$ Physics and Astronomy Department, Stony Brook University, Stony Brook, NY 11794, USA

${ }^{24}$ Department of Chemistry and Physics, Purdue University Calumet, Hammond, IN 46323-2094, USA

${ }^{25}$ Istituto Nazionale di Fisica Nucleare, Sezione di Trieste, and Università di Trieste, I-34127 Trieste, Italy

${ }^{26}$ Laboratory for Astroparticle Physics, University of Nova Gorica, Vipavska 13, SI-5000 Nova Gorica, Slovenia

${ }^{27}$ Cerro Tololo Inter-American Observatory, National Optical Astronomy Observatory, Casilla 603, La Serena, Chile

${ }^{28}$ Space Telescope Science Institute, 3700 San Martin Drive, Baltimore, MD 21218, USA

${ }^{29}$ Department of Physics, University of Surrey, Guildford GU2 7XH, UK

${ }^{30}$ Laboratório Interinstitucional de e-Astronomia-LIneA, Rua Gal. José Cristino 77, Rio de Janeiro, RJ-20921-400, Brazil

${ }^{31}$ Institut de Ciències de l'Espai, IEEC-CSIC, Campus UAB, Facultat de Ciències, Torre C5 par-2, E-08193 Bellaterra, Barcelona, Spain

${ }^{32}$ Department of Physics \& Astronomy, University College London, Gower Street, London, WC1E 6BT, UK

${ }^{33}$ Carnegie Observatories, 813 Santa Barbara Street, Pasadena, CA 91101, USA

${ }^{34}$ Department of Physics and Astronomy, University of Pennsylvania, Philadelphia, PA 19104, USA

${ }^{35}$ Institut d'Astrophysique de Paris, Univ. Pierre et Marie Curie \& CNRS UMR7095, F-75014 Paris, France

${ }^{36}$ SLAC National Accelerator Laboratory, Menlo Park, CA 94025, USA

${ }^{37}$ Observatório Nacional, Rua Gal. José Cristino 77, Rio de Janeiro, RJ-20921-400, Brazil

${ }^{38}$ National Center for Supercomputing Applications, 1205 West Clark Street, Urbana, IL 61801, USA

${ }^{39}$ Institute of Cosmology \& Gravitation, University of Portsmouth, Portsmouth, PO1 3FX, UK 


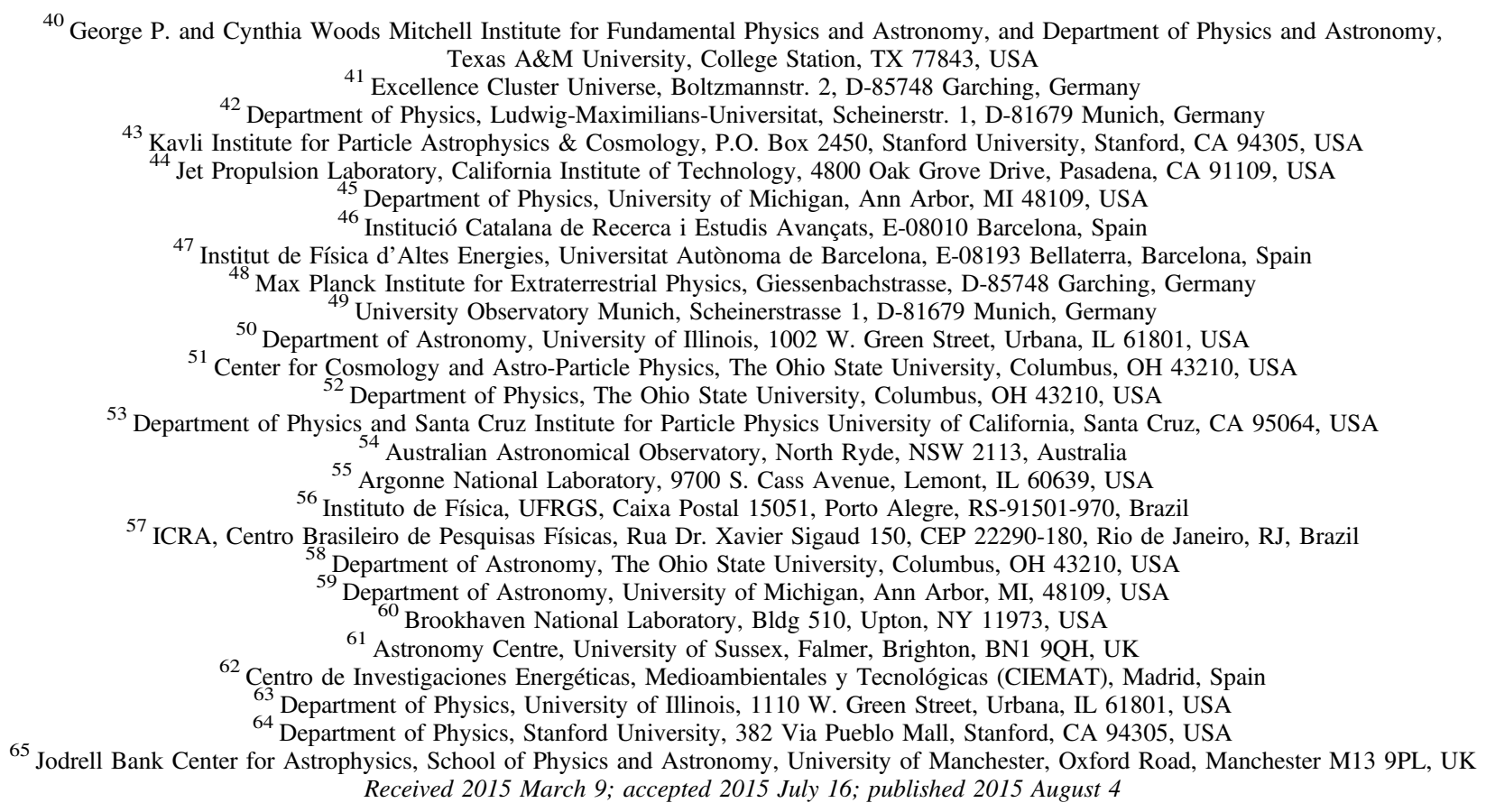

\section{ABSTRACT}

Due to their proximity, high dark-matter (DM) content, and apparent absence of non-thermal processes, Milky Way dwarf spheroidal satellite galaxies (dSphs) are excellent targets for the indirect detection of DM. Recently, eight new dSph candidates were discovered using the first year of data from the Dark Energy Survey (DES). We searched for gamma-ray emission coincident with the positions of these new objects in six years of Fermi Large Area Telescope data. We found no significant excesses of gamma-ray emission. Under the assumption that the DES candidates are dSphs with DM halo properties similar to the known dSphs, we computed individual and combined limits on the velocity-averaged DM annihilation cross section for these new targets. If the estimated DM content of these dSph candidates is confirmed, they will constrain the annihilation cross section to lie below the thermal relic cross section for DM particles with masses $\lesssim 20 \mathrm{GeV}$ annihilating via the $b \bar{b}$ or $\tau^{+} \tau^{-}$channels.

Key words: dark matter - galaxies: dwarf - gamma rays: galaxies - Local Group

\section{INTRODUCTION}

In the standard model of cosmology, dark matter (DM) is the dominant component of matter in the universe. Weakly interacting massive particles (WIMPs) are an attractive candidate to constitute some or all of DM (e.g., Bertone et al. 2005; Feng 2010). If WIMPs are in thermal equilibrium in the early universe and have a velocity-averaged annihilation cross section of $\langle\sigma v\rangle \sim 2.2 \times 10^{-26} \mathrm{~cm}^{3} \mathrm{~s}^{-1}$, their relic abundance can account for the observed DM abundance measured today (e.g., Steigman et al. 2012). WIMPs may continue to annihilate in regions of high DM density to produce energetic Standard Model particles that can be detected as indirect signatures of DM. These indirect searches complement terrestrial searches for DM in accelerator and direct detection experiments (e.g., Bauer et al. 2015).

Gamma rays are one product of WIMP annihilations (Baltz et al. 2008; Bringmann \& Weniger 2012); they may be produced directly or in a shower of secondary particles. Depending on the WIMP mass, these gamma-rays could be detectable with the Fermi Large Area Telescope (LAT) (Atwood et al. 2009).

\footnotetext{
${ }^{66}$ Royal Swedish Academy of Sciences Research Fellow, funded by a grant from the K. A. Wallenberg Foundation.

${ }^{67}$ NASA Postdoctoral Program Fellow, USA.
}

The integrated gamma-ray flux in a specific energy range $\left(E_{\min }<E<E_{\max }\right)$ and region of interest (ROI) on the sky from DM annihilation is given by

$$
\begin{aligned}
\varphi_{s}(\Delta \Omega)= & \underbrace{\frac{1}{4 \pi} \frac{\langle\sigma v\rangle}{2 m_{\mathrm{DM}}^{2}} \int_{E_{\min }}^{E_{\max }} \frac{d N_{\gamma}}{d E_{\gamma}} d E_{\gamma}}_{\text {particle physics }} \\
& \times \underbrace{\int_{\Delta \Omega} \int_{\mathrm{LOS}} \rho_{\mathrm{DM}}^{2}(\boldsymbol{r}) d s d \Omega}_{J \text {-factor }},
\end{aligned}
$$

where the first term encompasses the particle properties of the DM, while the second term (the so-called " $J$-factor") incorporates information about the distribution of DM along the line of sight. Specifically, $m_{\mathrm{DM}}$ is the DM particle mass, $d N_{\gamma} / d E_{\gamma}$ is the differential gamma-ray yield per annihilation summed over all final states, $\Delta \Omega$ is the solid angle of the ROI, and $\rho_{\mathrm{DM}}(\boldsymbol{r})$ is the DM density.

Current $\mathrm{N}$-body cosmological simulations of Milky Waysized regions predict the existence of thousands of Galactic DM overdensities called subhalos (Diemand et al. 2008; Springel et al. 2008). Luminous Milky Way dwarf spheroidal satellite galaxies (dSphs) are believed to reside in a subset of the most massive subhalos. The Milky Way dSphs are especially 
promising targets for indirect DM searches due to their large DM content, low diffuse Galactic $\gamma$-ray foregrounds, and lack of conventional astrophysical $\gamma$-ray production mechanisms (McConnachie 2012). Several searches for gamma-ray emission from known dSphs have been performed using LAT data, none of which has resulted in a positive detection (e.g., Abdo et al. 2010; Ackermann et al. 2011, 2014, 2015a; GeringerSameth \& Koushiappas 2011; Mazziotta et al. 2012; GeringerSameth et al. 2015b).

The census of known Milky Way satellites is certainly incomplete. Prior to the Sloan Digital Sky Survey (SDSS) (York et al. 2000), there were ten dSphs known to orbit the Milky Way (called classical dwarfs). The deep and systematic coverage of the northern celestial hemisphere by SDSS has more than doubled the number of known Milky Way satellites (McConnachie 2012). Additionally, SDSS data led to the discovery of a new population of "ultra-faint" satellite galaxies, which were found to be the most DM dominated objects known (Simon \& Geha 2007; Strigari et al. 2008b; Geha et al. 2009). The Dark Energy Survey (DES; Abbott et al. 2005) is a southernhemisphere optical survey expected to find new dSphs (Tollerud et al. 2008; Hargis et al. 2014), which would increase the sensitivity of searches for particle DM (He et al. 2015).

Photometric survey data can be used to identify stellar overdensities associated with satellite dwarf galaxies or globular clusters. Satellite galaxies require DM to explain their observed kinematics, while the mass of globular clusters can be accounted for by their visible matter alone. Globular clusters can be distinguished from dwarf galaxies based on spectroscopic measurements (Willman \& Strader 2012). The range of stellar metallicities in globular clusters is narrower than that observed in dSph galaxies. Though globular clusters and satellite galaxies may possess similar stellar velocity dispersions, the larger spatial extent of dwarf galaxies implies that they are DM-dominated.

The first internal annual release of DES data (Y1A1) covers $\sim 1800 \mathrm{deg}^{2}$ in the southern hemisphere $\left(\sim 1600 \mathrm{deg}^{2}\right.$ not overlapping with SDSS). ${ }^{68}$ Recent studies of the Y1A1 data set have revealed eight new dSph candidates (Bechtol et al. 2015; Koposov et al. 2015). ${ }^{69}$ Since the LAT continuously surveys the entire sky, LAT data collected over the duration of the mission can be used to search for gamma-ray emission from the DES dSph candidates.

\section{DISCOVERY OF NEW dSph CANDIDATES WITH DES}

Current and near-future deep wide-field optical imaging surveys have the potential to discover many new ultra-faint Milky Way satellites (Tollerud et al. 2008; He et al. 2015; Hargis et al. 2014). The ensemble of PanSTARRS (Kaiser et al. 2002), the SkyMapper Southern Sky Survey (Keller et al. 2007), DES (Abbott et al. 2005), and the Large Synoptic Survey Telescope (Ivezic et al. 2008) will explore large areas of the sky to unprecedented depths. Here, we focus on a set of $\mathrm{dSph}$ candidates recently found in first-year DES data.

Details regarding the first-year DES data set and techniques to search for ultra-faint dSphs are provided in Bechtol et al. (2015) and Koposov et al. (2015). Briefly, a dSph candidate is identified as a statistically significant arcminute-scale

\footnotetext{
68 http://data.darkenergysurvey.org/aux/releasenotes/DESDMrelease.html

69 Koposov et al. (2015) find a ninth candidate inside the DES year-one imaging footprint but outside the Y1A1 coadd catalog.
}

Table 1

DES dSph Candidates and Estimated $J$-factors

\begin{tabular}{lccc}
\hline \hline Name & $\begin{array}{c}(\ell, b)^{\mathrm{a}} \\
(\mathrm{deg})\end{array}$ & $\begin{array}{c}\text { Distance }^{\mathrm{b}} \\
(\mathrm{kpc})\end{array}$ & $\begin{array}{c}\log _{10}(\text { Est. J })^{\mathrm{c}} \\
\log _{10}\left(\mathrm{GeV}^{2} \mathrm{~cm}^{-5}\right)\end{array}$ \\
\hline DES J0222.7-5217 & $(275.0,-59.6)$ & 95 & 18.3 \\
DES J0255.4-5406 & $(271.4,-54.7)$ & 87 & 18.4 \\
DES J0335.6-5403 & $(266.3,-49.7)$ & 32 & 19.3 \\
DES J0344.3-4331 & $(249.8,-51.6)$ & 330 & 17.3 \\
DES J0443.8-5017 & $(257.3,-40.6)$ & 126 & 18.1 \\
DES J2108.8-5109 & $(347.2,-42.1)$ & 69 & 18.6 \\
DES J2251.2-5836 & $(328.0,-52.4)$ & 58 & 18.8 \\
DES J2339.9-5424 & $(323.7,-59.7)$ & 95 & 18.3 \\
\hline
\end{tabular}

Notes.

${ }^{a}$ Galactic longitude and latitude.

${ }^{\mathrm{b}}$ We note that typical uncertainties on the distances of dSphs are $10 \%-15 \%$.

${ }^{c} J$-factors are calculated over a solid angle of $\Delta \Omega \sim 2.4 \times 10^{-4}$ sr (angular radius 0.5 ). See text for more details.

overdensity of resolved stars consistent with an old ( $>10 \mathrm{Gyr})$ and metal-poor $(Z \sim 0.0002)$ stellar population. A variety of search techniques have been applied to the first-year DES data, including visual inspection of DES images, thresholding stellar density maps, scanning with optimized spatial filters, and automated matched-filter maximum-likelihood algorithms. The physical characteristics of dSph candidates (e.g., centroid position, distance, and spatial extension) can be inferred by fitting the spatial and color-magnitude distributions of the stars. Table 1 provides a summary of the eight dSph candidates reported by Bechtol et al. (2015).

\section{LAT ANALYSIS}

To search for gamma-ray emission from these new dSph candidates, we used six years of LAT data (2008 August 4 to 2014 August 5) passing the P8R2_SOURCE event class selections from $500 \mathrm{MeV}$ to $500 \mathrm{GeV}$. The low-energy bound of $500 \mathrm{MeV}$ is selected to mitigate the impact of leakage from the bright limb of the Earth because the point-spread function (PSF) broadens considerably below that energy. The highenergy bound of $500 \mathrm{GeV}$ is chosen to mitigate the effect of the increasing residual charged-particle background at higher energies (Ackermann et al. 2015b). Compared to the previous iteration of the LAT event-level analysis, Pass 8 provides significant improvements in all areas of LAT analysis; specifically the differential point-source sensitivity improves by $\sim 30 \%-50 \%$ in $\mathrm{P} 8 \mathrm{R} 2 \_$SOURCE_V6 relative to P7REP_SOURCE_V15 (Atwood et al. 2013). To remove gamma-rays produced by cosmic-ray interactions in the Earth's limb, we rejected events with zenith angles greater than $100^{\circ}$. Additionally, events from time intervals around bright gammaray bursts and solar flares were removed using the same method as in the 4-year catalog analysis (3FGL; Acero et al. 2015). To analyze the $\mathrm{dSph}$ candidates in Table 1 , we used $10^{\circ} \times 10^{\circ}$ ROIs centered on each object. Data reduction was performed using ScienceTools version 10-01-00. ${ }^{70}$ Figure 1 shows smoothed counts maps around each candidate for energies $>1 \mathrm{GeV}$. The candidate $\mathrm{dSphs}$ reside in regions of the sky where the diffuse background is relatively smooth. With the exception of DES J0255.4-5406, all the objects are located

\footnotetext{
${ }^{70}$ http://fermi.gsfc.nasa.gov/ssc/data/analysis/software/
} 

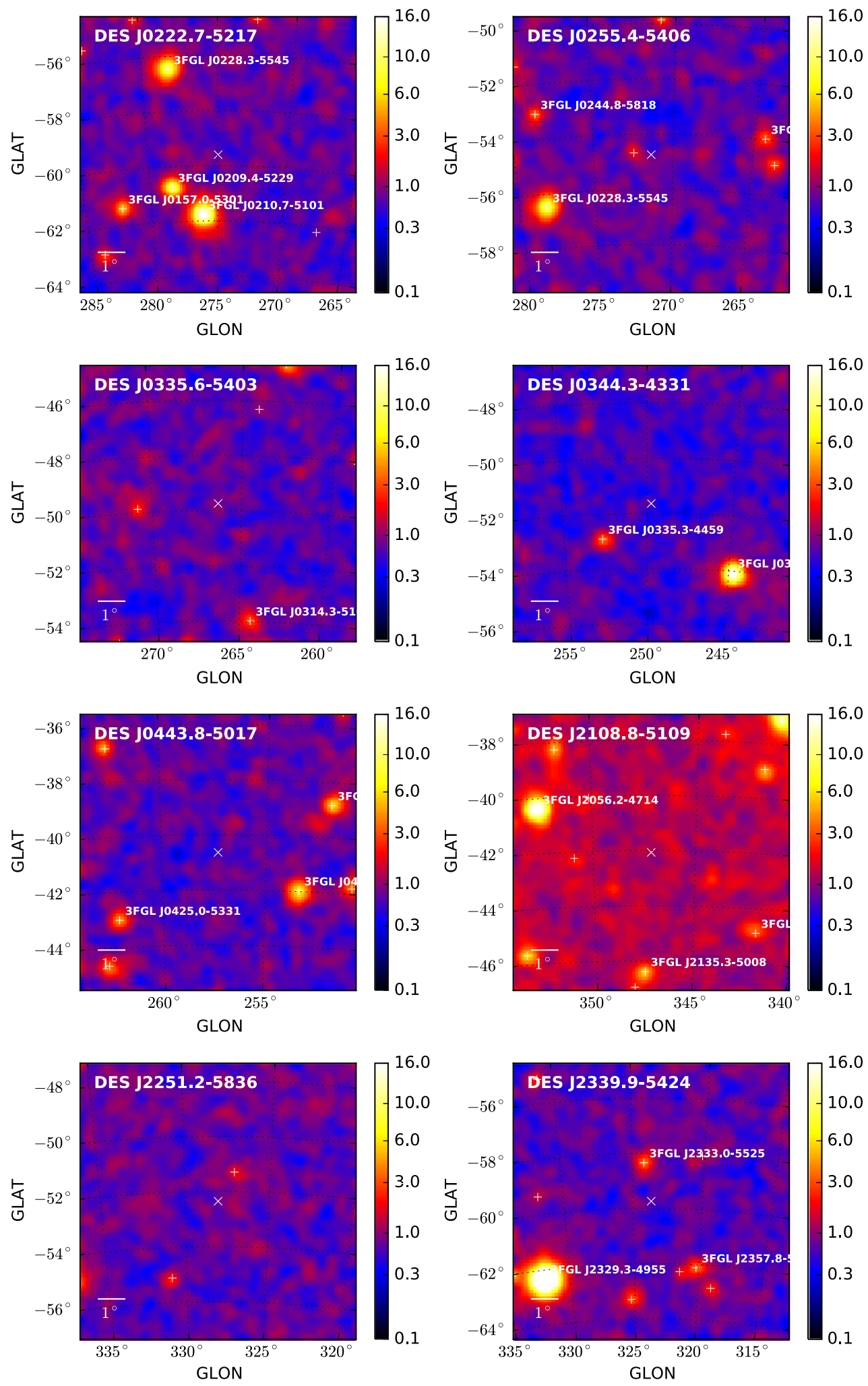

Figure 1. LAT counts maps in $10^{\circ} \times 10^{\circ}$ ROI centered at each DES dSph candidate (white " $\times$ " symbols), for $E>1 \mathrm{GeV}$, smoothed with a $0.25 \mathrm{Gaussian}$ kernel. All 3FGL sources in the ROI are indicated with white "+" symbols, and those with TS $>100$ are explicitly labeled.

more than $1^{\circ}$ from 3FGL background sources (DES J0255.4 -5406 is located 0.63 from 3FGL J0253.1-5438).

We applied the search procedure presented in Ackermann et al. (2015a) to the new DES dSph candidates. Specifically, we performed a binned maximum-likelihood analysis in 24 logarithmically spaced energy bins and $0: 1$ spatial pixels. Data are additionally partitioned in one of four PSF event types, which are combined in a joint-likelihood function when performing the fit to each ROI (Ackermann et al. 2015a). 


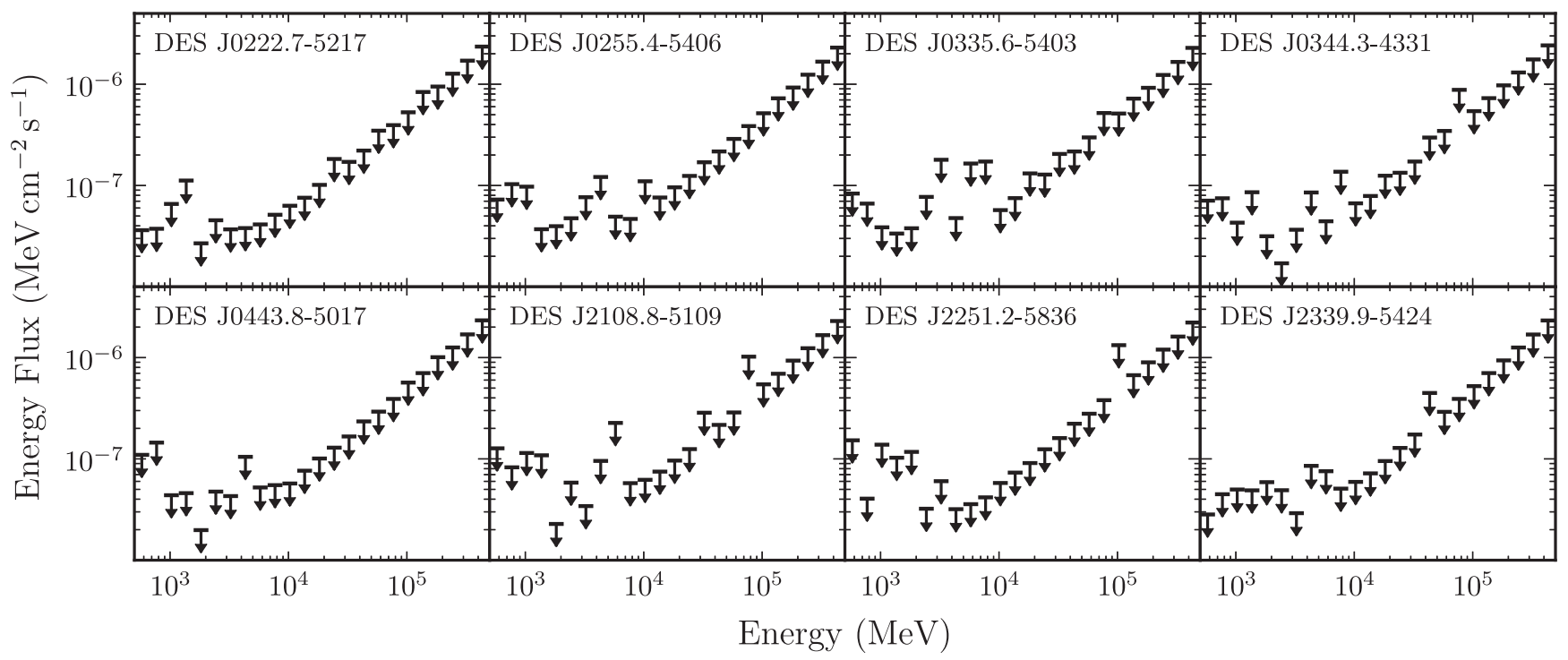

Figure 2. Bin-by-bin integrated energy-flux upper limits at 95\% confidence level for the eight DES dSph candidates modeled as point-like sources.

We used a diffuse emission model based on the model for Galactic diffuse emission derived from an all-sky fit to the Pass 7 Reprocessed data, ${ }^{71}$ but with a small $(<10 \%)$ energy-dependent correction to account for differences in the Pass 8 instrument response. ${ }^{72}$ Additionally, we model extragalactic gamma-ray emission and residual charged particle contamination with an isotropic model fit to the Pass 8 data. These models will be included in the forthcoming public Pass 8 data release. Point-like sources from the recent 3FGL catalog (Acero et al. 2015) within $15^{\circ}$ of the ROI center were also included in the ROI model. The spectral parameters of these sources were fixed at their 3FGL catalog values. The flux normalizations of the Galactic diffuse and isotropic components and 3FGL catalog sources within the $10^{\circ} \times 10^{\circ} \mathrm{ROI}$ were fit simultaneously in a binned likelihood analysis over the broadband energy range from $500 \mathrm{MeV}$ to $500 \mathrm{GeV}$. The fluxes and normalizations of the background sources are insensitive to the inclusion of a putative power-law source at the locations of the DES dSph candidates, as expected when there is no bright point source at the center of the ROI.

In contrast to Ackermann et al. (2015a), we modeled the dSph candidates as point-like sources rather than spatially extended Navarro, Frenk and White (NFW) DM density profiles (Navarro et al. 1997). This choice was motivated by the current uncertainty in the spatial extension of the DM halos of these new objects. Previous studies have shown that the LAT flux limits are fairly insensitive to modeling $\mathrm{dSph}$ targets as point-like versus spatially extended sources (Ackermann et al. 2014). Following the procedure of Ackermann et al. (2015a), we fit for excess gamma-ray emission associated with each target in each energy bin separately to derive flux constraints that are independent of the choice of spectral model. Within each bin, we model the putative dSph source with a power-law spectral model $\left(d N / d E \propto E^{-\Gamma}\right)$ with spectral index

\footnotetext{
$\overline{71}$ http://fermi.gsfc.nasa.gov/ssc/data/access/lat/BackgroundModels.html

72 Standard LAT analyses treat the diffuse emission model as being defined in terms of true energy, but the model was necessarily derived from the measured energies of events. This implies a weak dependence of the model on the instrument response functions. The correction applied to the diffuse emission model accounts for the different energy dependence of the effective area and energy resolution between Pass 7 Reprocessed and Pass 8.
}

of $\Gamma=2$. We show the bin-by-bin integrated energy-flux $95 \%$ confidence level upper limits for each $\mathrm{dSph}$ candidate in Figure 2. The Poisson likelihoods from each bin were combined to form global spectral likelihoods for different DM annihilation channels and masses.

We tested for excess gamma-ray emission consistent with two representative DM annihilation channels (i.e., $b \bar{b}$ and $\tau^{+} \tau^{-}$) and a range of particle masses from $2 \mathrm{GeV}$ to $10 \mathrm{TeV}$ (when kinematically allowed). No significant excess gammaray emission was observed from any of the DES dSph candidates for any of the DM masses or channels tested. The data were found to be well described by the background model with no significant residuals observed.

We calculated the test statistic (TS) for signal detection by comparing the likelihood values both with and without the added dSph candidate template (see Equation (6) in Ackermann et al. 2015a).

The most significant excess, $\mathrm{TS}=6.8$, was for DES J0335.6 -5403 and a DM particle with $m_{\mathrm{DM}}=25 \mathrm{GeV}$ annihilating into $\tau^{+} \tau^{-}$. ${ }^{73}$ To convert from TS to a local $p$-value, we use the TS distribution measured by performing our search for gammaray emission in 4000 random blank sky fields (Ackermann et al. 2014, 2015a). ${ }^{74}$ We find that TS $=6.8$ corresponds to a local significance of $2.4 \sigma(p=0.01)$. After applying a trials factor to account for our scan in mass and annihilation channel, we calculate a significance of $1.65 \sigma(p=0.05)$ for this target. The global significance when accounting for fitting eight target locations is $0.43 \sigma(p=0.33)$.

Following the procedure described in the supplemental material of Ackermann et al. (2015a), we investigated the systematic uncertainties related to uncertainties in the diffuse emission model by refitting with eight alternative diffuse

\footnotetext{
73 We note that the radio-continuum source PMN J0335-5406 is located $\sim 0$. 1 from the center of DES J0335.6-5403. It is not a cataloged blazar, but has radio and infrared spectral characteristics consistent with blazars detected by the LAT.

${ }_{74}$ Though our blank sky ROIs are not independent, the overlap is negligible since we are testing for a point source at the center of the ROI. We have verified with a Monte Carlo all-sky realization that the TS distribution from our blank-sky analysis follows the asymptotic expectation when the background model perfectly describes the data.
} 


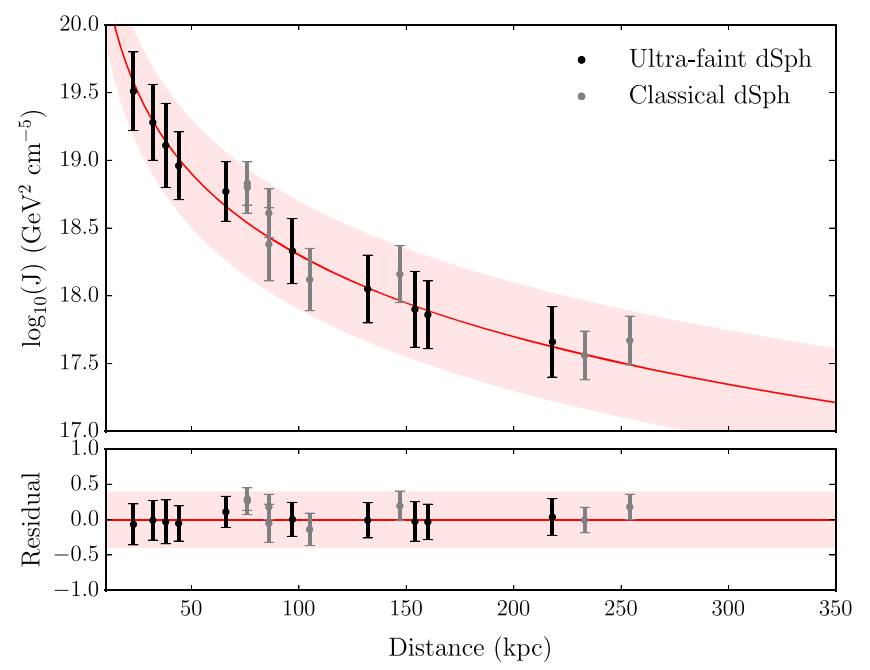

Figure 3. $J$-factor distance scaling. Black points are from Table 1 in Ackermann et al. (2014). The red curve is our best fit with an assumed inverse square distance relation (see the text). The red band shows the $\pm 0.4 \mathrm{dex}$ uncertainty that we adopt.

models (de Palma et al. 2013). We found that using the alternative diffuse models varied the calculated limits and TS values by $\lesssim 20 \%$.

\section{ESTIMATING J-FACTORS FOR THE DES dSph CANDIDATES}

The DM content of the DES dSph candidates cannot be determined without spectroscopic observations of their member stars. However, it is possible to predict the upper limits on the DM annihilation cross section that would be obtained given such observations by making the assumption that these candidates possess DM distributions similar to the known $\mathrm{dSphs}$. Our estimates for the astrophysical $J$-factors of these candidates are motivated by two established relationships. First, the known dSphs have a common mass scale in their interiors, roughly $10^{7} M_{\odot}$ within their central $300 \mathrm{pc}$ (Strigari et al. 2008a). This radius is representative of the half light radius for classical dSphs, but is outside the visible stellar distribution of several ultra-faint satellites. More generally, the half-light radius of a dSph and the mass within the half-light radius have been found to obey a simple scaling relation, assuming that the velocity dispersions are nearly constant in radius and the anisotropy of the stars is not strongly radially dependent (Walker et al. 2009; Wolf et al. 2010).

In the analysis that follows, we used the ten ultra-faint SDSS satellites with spectroscopically determined $J$-factors as a representative set of known dSphs. Specifically, we take the $J$-factors calculated assuming an NFW profile integrated over a radius of 0.5 for Boötes I, Canes Venatici I, Canes Venatici II, Coma Berenices, Hercules, Leo IV, Segue 1, Ursa Major I, Ursa Major II, and Willman 1 (see Table 1 in Ackermann et al. 2014). Figure 3 shows the relation between the heliocentric distances and $J$-factors of ultra-faint and classical dSphs. As expected from their similar interior DM masses, the $J$-factors of the known dSphs scale approximately as the inverse square of the distance. The best-fit normalization is

\footnotetext{
${ }^{75}$ When using the values derived by Geringer-Sameth et al. (2015a) and including Segue 2, we find a best-fit normalization of $\log _{10}(J)=18.0 \pm 0.1$ at $d=100 \mathrm{kpc}$.
}

$\log _{10}(J)=18.3 \pm 0.1$ at $d=100 \mathrm{kpc}$. We obtain a similar best-fit value, $\log _{10}(J)=18.1 \pm 0.1$ at $d=100 \mathrm{kpc}$, using the $J$-factors derived by Geringer-Sameth et al. (2015a), who assumed a generalized NFW profile and omitted Willman $1 .^{75}$ We note that the limited scatter in Figure 3 is primarily due to the known dSphs residing in similar DM halos (Ackermann et al. 2014). Under the assumption that the new DES $\mathrm{dSph}$ candidates belong to the same population, we estimated their $J$-factors based on the distances derived from the DES photometry. Table 1 gives the estimated $J$-factors integrated over a solid-angle of $\Delta \Omega \sim 2.4 \times 10^{-4}$ sr using our simple, empirical relation.

Several caveats should be noted. None of the DES candidates have been confirmed to be gravitationally bound. It is possible that some have stellar populations characteristic of galaxies but lack substantial DM content, as is the case for Segue 2 (Kirby et al. 2013), or have complicated kinematics that are difficult to interpret (Willman et al. 2011). Further, some of the M31 dSphs have been found to deviate from these relations, though it is possible that these deviations are due to tidal disruption (Collins et al. 2014). Kinematic measurements of the member stars are needed to unambiguously resolve these questions.

Using the $J$-factor estimates presented in Table 1, we followed the likelihood procedure detailed in Ackermann et al. (2015a) to obtain limits on DM annihilation from these eight candidates shown in Figure 4.

We assumed a symmetric logarithmic uncertainty on the $J$-factor of \pm 0.4 dex for each DES candidate. This value is representative of the uncertainties from ultra-faint dSphs (Ackermann et al. 2011; Geringer-Sameth et al. 2015a) and is somewhat larger than the uncertainties derived in Martinez (2015). The \pm 0.4 dex uncertainty is intended to represent the expected measurement uncertainty on the $J$-factors of the DES candidates after kinematic follow up. The corresponding uncertainty band is illustrated in Figure 3. We apply the same methodology as Ackermann et al. (2015a) to account for the $J$-factor uncertainty on each DES candidate by modeling it as a $\log$ normal distribution with $J_{\text {obs }, i}$ equal to the values in Table 1, and $\sigma_{i}=0.4$ dex (see Equation (3) of Ackermann et al. 2015a).

We derived individual and combined limits on the DM annihilation cross section for DM annihilation via the $b \bar{b}$ and $\tau^{+} \tau^{-}$channels, under the assumption that each DES candidate is a dSph and has the $J$-factor listed in Table 1 . We note that when using a $J$-factor uncertainty of \pm 0.6 dex instead of \pm 0.4 dex, the individual dwarf candidate limits worsen by a factor of $\sim 1.6$, while the combined limits worsen by $15 \%-20 \%$. We stress that the distance-estimated limits may differ substantially as spectroscopic data become available to more robustly constrain the DM content of the DES candidates. However, once measured $J$ factors are obtained, the observed limits from each candidate will scale linearly with the measured $J$-factor relative to our estimates. Given the current uncertainty regarding the nature of the dSph candidates, we do not combine limits with those from previously known dSphs (i.e., Ackermann et al. 2015a).

\section{DISCUSSION AND CONCLUSIONS}

The discovery of eight $\mathrm{dSph}$ candidates in the first year of DES observations sets an optimistic tone for future $\mathrm{dSph}$ detections from DES and other optical surveys. DES J0335.6-5403, at a distance of $\sim 32 \mathrm{kpc}$, is a particularly interesting candidate in this context, and should be considered a 

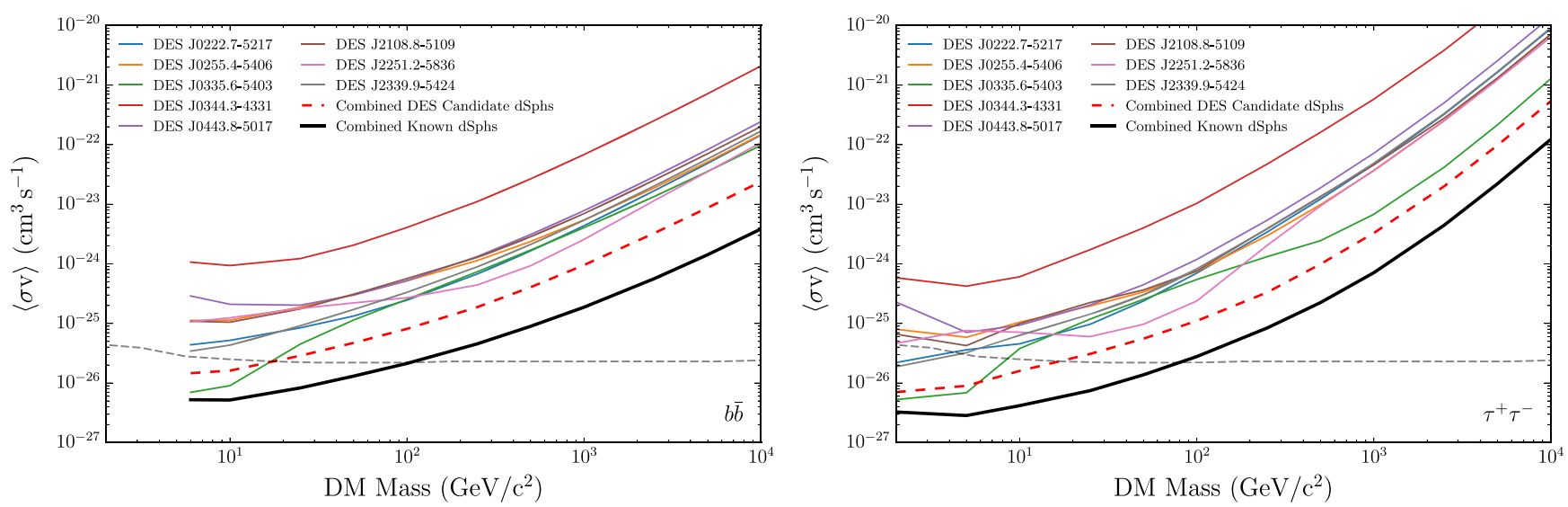

Figure 4. Upper limits on the velocity-averaged DM annihilation cross section at 95\% confidence level for DM annihilation to $b \bar{b}$ (left) and $\tau^{+} \tau^{-}$(right) derived using distance-estimated $J$-factors. Individual limits for each DES candidate dSph, as well as the combined limits (dashed red line) from the eight new candidates are shown. Here we assume that each candidate is a dSph and that future kinematic analyses will confirm the $J$-factors estimated based on photometric data (see the text). For reference, we show the current best limits derived from a joint analysis of 15 previously known dSphs with kinematically constrained $J$-factors (black curve) (Ackermann et al. 2015a). The dashed gray curve shows the thermal relic cross section derived by Steigman et al. (2012).

high-priority target for spectroscopic follow up. The location of any newly discovered $\mathrm{dSph}$, including the candidates investigated in this work, will have already been regularly observed since the beginning of the Fermi mission. No significant gamma-ray excess was found coincident with any of the eight new DES dSph candidates considered here. If kinematic analyses find the $\mathrm{dSph}$ candidates to have $J$-factors similar to our estimates, they constrain the annihilation cross section to lie below the thermal relic cross section for DM particles with masses $\lesssim 20 \mathrm{GeV}$ annihilating via the $b \bar{b}$ or $\tau^{+} \tau^{-}$channels.

The population of nearby DM-dominated dSphs represents an independent set of targets to test possible signals of DM annihilation in other regions such as the Galactic center (e.g., Gordon \& Macias 2013; Abazajian et al 2014; Calore et al. 2015; Daylan et al. 2014). Though the expected DM signals of individual dSphs are smaller than that of the Galactic center, a joint-likelihood analysis of many dSphs can probe the DM annihilation cross section at a similar level of sensitivity. The incorporation of new dSphs in indirect searches for DM with the LAT will further enhance the sensitivity of this method.

Independent analyses of DES J0335.6-5403 have been performed by Geringer-Sameth et al. (2015c) and Hooper \& Linden (2015). While the analysis details differ (e.g., the data set, the search technique, statistical methodology, and the calculation of the trials factor), each analysis finds the largest TS value in the direction of DES J0335.6-5403. The $p$-values derived in Geringer-Sameth et al. and Hooper \& Linden are smaller than those found in this work. One key difference is that Geringer-Sameth et al. and Hooper \& Linden use the publicly available Pass 7 Reprocessed data, while the analysis presented here uses the soon-to-be-released Pass 8 data, which improves the point-source sensitivity by $\sim 30 \%-$ $50 \%$ in the relevant energy range.

The Fermi-LAT Collaboration acknowledges support for LAT development, operation and data analysis from NASA and DOE (United States), CEA/Irfu and IN2P3/CNRS (France), ASI and INFN (Italy), MEXT, KEK, and JAXA (Japan), and the K. A. Wallenberg Foundation, the Swedish Research Council and the National Space Board (Sweden). Science analysis support in the operations phase from INAF (Italy) and CNES (France) is also gratefully acknowledged.
Funding for the DES Projects has been provided by the U.S. Department of Energy, the U.S. National Science Foundation, the Ministry of Science and Education of Spain, the Science and Technology Facilities Council of the United Kingdom, the Higher Education Funding Council for England, the National Center for Supercomputing Applications at the University of Illinois at Urbana-Champaign, the Kavli Institute of Cosmological Physics at the University of Chicago, the Center for Cosmology and Astro-Particle Physics at the Ohio State University, the Mitchell Institute for Fundamental Physics and Astronomy at Texas A\&M University, Financiadora de Estudos e Projetos, Fundação Carlos Chagas Filho de Amparo à Pesquisa do Estado do Rio de Janeiro, Conselho Nacional de Desenvolvimento Científico e Tecnológico and the Ministério da Ciência, Tecnologia e Inovação, the Deutsche Forschungsgemeinschaft and the Collaborating Institutions in the Dark Energy Survey. The DES data management system is supported by the National Science Foundation under Grant Number AST-1138766. The DES participants from Spanish institutions are partially supported by MINECO under grants AYA2012-39559, ESP2013-48274, FPA2013-47986, and Centro de Excelencia Severo Ochoa SEV-2012-0234, some of which include ERDF funds from the European Union. The Collaborating Institutions are Argonne National Laboratory, the University of California at Santa Cruz, the University of Cambridge, Centro de Investigaciones Enérgeticas, Medioambientales y Tecnológicas-Madrid, the University of Chicago, University College London, the DES-Brazil Consortium, the University of Edinburgh, the Eidgenössische Technische Hochschule (ETH) Zürich, Fermi National Accelerator Laboratory, the University of Illinois at Urbana-Champaign, the Institut de Ciències de l'Espai (IEEC/CSIC), the Institut de Física d'Altes Energies, Lawrence Berkeley National Laboratory, the Ludwig-Maximilians Universität München and the associated Excellence Cluster Universe, the University of Michigan, the National Optical Astronomy Observatory, the University of Nottingham, the Ohio State University, the University of Pennsylvania, the University of Portsmouth, SLAC National Accelerator Laboratory, Stanford University, the University of Sussex, and Texas A\&M University. A.C.R. acknowledges financial support provided by the PAPDRJ CAPES/FAPERJ Fellowship. A.A.P. was supported by DOE 
grant DE-AC02-98CH10886 and by JPL, run by Caltech under a contract for NASA. This research has made use of the NASA/IPAC Extragalactic Database (NED) which is operated by the Jet Propulsion Laboratory, California Institute of Technology, under contract with the National Aeronautics and Space Administration. We would like to thank the anonomous referee for many helpful comments.

Facilities: Blanco, Fermi-LAT

\section{REFERENCES}

Abazajian, K. N., Canac, N., Horiuchi, S., \& Kaplinghat, M. 2014, PhRvD, 90, 023526

Abbott, T., Aldering, G., Annis, J., et al. 2005, arXiv:astro-ph/0510346

Abdo, A. A., Ackermann, M., Ajello, et al. 2010, ApJ, 712, 147

Acero, F., Ackermann, M., Ajello, M., et al. 2015, arXiv:1501.02003

Ackermann, M., Ajello, M., Albert, A., et al. 2011, PhRvL, 107, 241302

Ackermann, M., Ajello, M., Albert, A., et al. 2015b, ApJ, 799, 86

Ackermann, M., Albert, A., Anderson, B., et al. 2014, PhRvD, 89, 042001

Ackermann, M., Albert, A., Anderson, B., et al. 2015a, arXiv:1503.02641

Atwood, W. B., Abdo, A. A., Ackermann, M., et al. 2009, ApJ, 697, 1071

Atwood, W., Albert, A., Baldini, L., et al. 2013, in 2012 Fermi Symposium

Proceedings, arXiv:1303.3514

Baltz, E., Berenji, B., Bertone, G., et al. 2008, JCAP, 0807, 013

Bauer, D., Buckley, J., Cahill-Rowley, M., et al. 2015, PDU, 7, 16

Bechtol, K., Drlica-Wagner, A., Balbinot, E., et al. 2015, ApJ, 807, 50

Bertone, G., Hooper, D., \& Silk, J. 2005, PhR, 405, 279

Bringmann, T., \& Weniger, C. 2012, PDU, 1, 194

Calore, F., Cholis, I., \& Weniger, C. 2015, JCAP, 03, 038

Collins, M. L., Chapman, S. C., Rich, R., et al. 2014, ApJ, 783, 7

Daylan, T., Finkbeiner, D. P., Hooper, D., et al. 2014, arXiv:1402.6703

de Palma, F., Brandt, T., Johannesson, G., \& Tibaldo, L. 2013, in 2012 Fermi Symposium Proceedings, arXiv:1304.1395

Diemand, J., Kuhlen, M., Madau, P., et al. 2008, Natur, 454, 735
Feng, J. L. 2010, ARA\&A, 48, 495

Geha, M., Willman, B., Simon, J. D., et al. 2009, ApJ, 692, 1464

Geringer-Sameth, A., \& Koushiappas, S. M. 2011, PhRvL, 107, 241303

Geringer-Sameth, A., Koushiappas, S. M., \& Walker, M. 2015a, ApJ, 801, 74

Geringer-Sameth, A., Koushiappas, S. M., \& Walker, M. G. 2015b, PhRvD, 91,083535

Geringer-Sameth, A., Walker, M. G., Koushiappas, S. M., et al. 2015c, PhRvL, in press (arXiv:1503.02320)

Gordon, C., \& Macias, O. 2013, PhRvD, 88, 083521

Hargis, J. R., Willman, B., \& Peter, A. H. G. 2014, ApJL, 795, L13

He, C., Bechtol, K., Hearin, A. P., \& Hooper, D. 2015, PhRvD, 91, 063515

Hooper, D., \& Linden, T. 2015, arXiv:1503.06209

Ivezic, Z., Tyson, J., Allsman, R., Andrew, J., \& Angel, R. 2008, arXiv:0805.2366

Kaiser, N., Aussel, H., Boesgaard, H., et al. 2002, Proc. SPIE, 4836, 154

Keller, S., Schmidt, B., \& Bessell, M. 2007, PASA, 24, 1

Kirby, E. N., Boylan-Kolchin, M., Cohen, J. G., et al. 2013, ApJ, 770, 16

Koposov, S. E., Belokurov, V., Torrealba, G., \& Evans, N. W. 2015, ApJ, 806,130

Martinez, G. D. 2015, MNRAS, 451, 2524

Mazziotta, M. N., Loparco, F., de Palma, F., \& Giglietto, N. 2012, Astropart., 37,26

McConnachie, A. W. 2012, AJ, 144, 4

Navarro, J. F., Frenk, C. S., \& White, S. D. 1997, ApJ, 490, 493

Simon, J. D., \& Geha, M. 2007, ApJ, 670, 313

Springel, V., Wang, J., Vogelsberger, M., et al. 2008, MNRAS, 391, 1685

Steigman, G., Dasgupta, B., \& Beacom, J. F. 2012, PhRvD, 86, 023506

Strigari, L. E., Bullock, J. S., Kaplinghat, M., et al. 2008a, Natur, 454, 1096

Strigari, L. E., Koushiappas, S. M., Bullock, J. S., et al. 2008b, ApJ, 678, 614

Tollerud, E. J., Bullock, J. S., Strigari, L. E., \& Willman, B. 2008, ApJ, 688,277

Walker, M. G., Mateo, M., Olszewski, E. W., et al. 2009, ApJ, 704, 1274

Willman, B., Geha, M., Strader, J., et al. 2011, AJ, 142, 128

Willman, B., \& Strader, J. 2012, AJ, 144, 76

Wolf, J., Martinez, G. D., Bullock, J. S., et al. 2010, MNRAS, 406, 1220

York, D. G., Adelman, J., Anderson, J. E., Jr., et al. 2000, AJ, 120, 1579 